\title{
Penultimate Unit Effects in Free-Radical Copolymerization. Number-Average Degree of Polymerization and Copolymerization Rate in Styrene/Methyl Methacrylate/Toluene System
}

\author{
Kousuke ONo and Shinya TeramaCHI \\ Department of Applied Chemistry, Faculty of Engineering, Kogakuin University, \\ 2665-1 Nakano-cho, Hachioji, Tokyo 192, Japan
}

(Received January 5, 1995)

\begin{abstract}
Recently, Fukuda et al. showed from the measurements of absolute rate constants that in the free-radical copolymerization the propagation step obeys the penultimate model and the termination step is diffusion-controlled. Also Fukuda et al. proposed the "radical stabilization energy model" in the free-radical copolymerization. To examine this copolymerization model, relationships between feed monomer composition $f_{1}$ and number-average degree of polymerization $X_{n}$ and between $f_{1}$ and copolymerization rate $R_{\mathrm{p}}$ were investigated for the copolymerization of styrene and methyl methacrylate in toluene at $40^{\circ} \mathrm{C}$. The composition of the copolymer $F_{1}$ was determined by ${ }^{1} \mathrm{H}$ NMR. The number-average molecular weight of the copolymer $M_{n}$ was measured to calculate $X_{n}$ by osmometry and size exclusion chromatography (SEC). By the so-called terminal model (the Mayo-Lewis model), the copolymer composition data was well described while the data of $X_{n}$ and $R_{\mathrm{p}}$ were not described. Alternatively, the model proposed by Fukuda et al. described well the composition data, the $X_{n}$ data and the $R_{\mathrm{p}}$ data.

KEY WORDS Diffusion-Controlled Termination / Terminal Model / Penultimate Model / Number-Average Degree of Polymerization /
\end{abstract}

In the free-radical copolymerization of many binary systems, the relationship between the monomer feed composition $f_{1}$ and the copolymer composition $F_{1}$ have been described by the Mayo-Lewis equation ${ }^{1}$ based on the terminal model.

$$
F_{1}=\frac{r_{1} f_{1}^{2}+f_{1} f_{2}}{r_{1} f_{1}^{2}+2 f_{1} f_{2}+r_{2} f_{2}^{2}}
$$

where $r_{1}$ and $r_{2}$ are the monomer reactivity ratios. From this fact, the free-radical copolymerization mechanism had been believed to obey the terminal model. Paradoxes in this model, however, had been indicated so far. For example, the values of cross termination factor $(\phi)$ are too large and dependent on the feed monomer composition.

Recently, Fukuda et al. showed that the Mayo-Lewis model fails to describe the relationship between the monomer feed composition and the absolute rate constant of propagation (when the rate of copolymerization is described as analogous to that of homopolymerization). Moreover they showed that the propagation reaction of the freeradical copolymerization obeys the penultimate model and the termination reaction is diffusion-controlled. ${ }^{2-9}$ The penultimate model with 8 rate constants has 6 rate constant ratios, which are defined by them as

$$
\begin{aligned}
& r_{1}=k_{111} / k_{112}, r_{2}=k_{222} / k_{221} \\
& r_{1}^{\prime}=k_{211} / k_{212}, r_{2}^{\prime}=k_{122} / k_{121} \\
& s_{1}=k_{211} / k_{111}, s_{2}=k_{122} / k_{222} \\
&\left(\sim \sim \sim \mathrm{M}_{i} \mathrm{M}_{j} \cdot+\mathrm{M}_{m} \stackrel{k_{i j m}}{\longrightarrow} \sim \sim \mathrm{M}_{i} \mathrm{M}_{j} \mathrm{M}_{m} \cdot\right)
\end{aligned}
$$




\section{Copolymerization Mechanism: Composition and $M_{n}$}

where $r_{i}$ 's are the monomer reactivity ratios and $s_{i}$ 's are reactivity ratios called "the radical reactivity ratio". In the case that the relationship between the monomer feed composition and the copolymer composition can be described by the Mayo-Lewis equation, $r_{i}=r_{i}^{\prime}$ is assumed. ${ }^{2}$ In this model, Fukuda et $a l$. presented "the radical stabilization energy model", 5 where it was assumed that by adding a monomer of species different from a terminal monomer (radical) to the terminal radical, the new terminal radical is stabilized, the reaction leading to the stabilization is fast $\left(r_{i}\right.$, small), and the stabilized radical reacts slowly $\left(s_{i}\right.$, small). This assumption gives a following equation.

$$
r_{1} r_{2}=s_{1} s_{2} \text {. }
$$

According to this new model presented by Fukuda et al., the penultimate unit effect does not appaer in composition but in rate constant of propagation. As the result, the composition curve of this model obeys the same as the Mayo-Lewis model, while the copolymerization rate and the number-avarage degree of polymerization is expected to obey the new model (penultimate model). In this study, styrene (S) and methyl methacrylate (MMA) were copolymerized in toluene. The copolymerization rate and the number-avarage degree of polymerization were estimated by membrane osmometry, dual detector size exclusion chromatography (SEC), and ${ }^{1} \mathrm{H}$ NMR to examine this new copolymerization model.

\section{EXPERIMENTAL}

\section{Copolymerization}

The monomers, S and MMA, were washed twice with $5 \%$ sodium hydroxide aqueous solution, followed by washing several times with saturated sodium chloride aqueous solution, and then dried over calcium chloride. The monomers were distilled under reduced nitrogen atmosphere before use. The initiator azobisisobutyronitrile was purified twice by recrystallization from the methanol solution. Toluene was purified by refluxing and distilling over sodiumwire under nitrogen atmosphere. The copolymerization was carried out at $40^{\circ} \mathrm{C}$ in toluene under the condition that the concentrations of the total monomers and the initiator were constant. The copolymerization was stopped by pouring the copolymerization mixture (ca. $200 \mathrm{ml}$ ) into methanol (ca. $2000 \mathrm{ml}$ ) as a precipitant. The yielded polymer was wawhed with methanol and dried in vacuo. The copolymer compositions were determined by ${ }^{1} \mathrm{H}$ NMR recorded on a JEOL EX-270 using $\mathrm{CDCl}_{3}$ as solvent at room temperature.

\section{Number-Average Degree of Polymerization}

The membrane osmometry was carried out at $20^{\circ} \mathrm{C}$ with Wescan Model 231 high speed membrane osmometry. Benzene was used as a solvent. The membrane used was regenerated cellulose (Wescan) of type RC52, pore size $0.010 \mu \mathrm{m}$, and lower limit of measurable molecular weight 30000 . Each samples were measured with four concentrations, ca. $2 \times$ $10^{-3} \mathrm{~g} \cdot \mathrm{cm}^{-3}, 4 \times 10^{-3} \mathrm{~g} \cdot \mathrm{cm}^{-3}, \quad 6 \times 10^{-3} \mathrm{~g} \cdot$ $\mathrm{cm}^{-3}$, and $8 \times 10^{-} 3 \mathrm{~g} \cdot \mathrm{cm}^{-3}$.

For the SEC measurements, two columns of TSK-GEL GMHHR-M (TOSOH), pump of CCPE (TOSOH), UV-detector of UV-8011 (TOSOH), RI-detector of RI-8 (TOSOH) and the thermostat of $\mathrm{CO}-8000$ (TOSOH) were used. The measurements were carrid out at $30^{\circ} \mathrm{C}$ by using distilled tetrahydrofuran (THF) as an eluent with the flow rate $1.0 \mathrm{~cm}^{3} \mathrm{~min}^{-1}$. The sample concentration was $0.2 \mathrm{w} / \mathrm{v} \%$ $(0.1 \mathrm{w} / \mathrm{v} \%$ for the standard polystyrenes), and the injection volume was $0.1 \mathrm{~cm}^{3}$. From the analysis of the chromatograms, the apparant number-average molecular weights (polystyrene standard) were calculated.

\section{RESULTS}

In Table I, data of copolymerization of $\mathrm{S}$ (subscript 1) and MMA (subscript 2) were shown. The conversions of all samples were 
Table I. (S/MMA) copolymerization in toluene at $40^{\circ} \mathrm{C}$

\begin{tabular}{|c|c|c|c|c|c|}
\hline \multirow{2}{*}{$f_{1}{ }^{\mathrm{a}}$} & {$[\mathrm{M}]^{\mathrm{b}}$} & Conversion & \multirow{2}{*}{$F_{1}^{\mathrm{c}}$} & \multirow{2}{*}{$10^{-5} M_{n}^{\mathrm{f}}$} & \multirow{2}{*}{$10^{-5} M_{n}{ }^{8}$} \\
\hline & $\mathrm{moll}^{-1}$ & $w t \%$ & & & \\
\hline 0.000 & 4.53 & 7.69 & 0.000 & 2.92 & 4.29 \\
\hline 0.050 & 4.53 & 4.02 & 0.118 & 1.80 & 2.97 \\
\hline 0.097 & 4.52 & 3.02 & 0.183 & 1.71 & 3.04 \\
\hline 0.200 & 4.53 & 2.12 & 0.297 & 1.66 & 1.94 \\
\hline 0.400 & 4.53 & 1.57 & 0.449 & 1.19 & 1.40 \\
\hline 0.750 & 4.53 & 1.31 & 0.695 & 0.860 & 1.02 \\
\hline 1.000 & 4.53 & 1.02 & 1.000 & 0.933 & 0.918 \\
\hline
\end{tabular}

a Mole fraction of styrene in feed. ${ }^{\mathrm{b}}$ Total monomer concentration. ${ }^{\mathrm{c}}$ AIBN concentration: $1.90 \times 10^{-2} \mathrm{moll}^{-1}$. ${ }^{\mathrm{d}}$ Reaction time, $180 \mathrm{~min}$. ${ }^{\mathrm{e}}$ Mole fraction of styrene in copolymer by ${ }^{1} \mathrm{H}$ NMR. ${ }^{\mathrm{f}} \mathrm{SEC}$ value (polystyrene standard). ${ }^{\mathrm{g}}$ Osmometry.

lower than $10 \mathrm{wt} \%$. As the $\mathrm{S}$ content in the copolymer $f_{1}$ increases, the number-average molecular weights $M_{n}$ 's measured by membrane osmometry and by SEC (polystyrene standard) decrease gradually. The difference of both $M_{n}$ 's becomes large, as the $\mathrm{S}$ content decrease. The increasing difference of $M_{n}$ by SEC from the value by osmometry may be ascribed to the dependency of molecular size in solution on the copolymer composition. For the polystyrene sample $\left(f_{1}=1\right)$, both $M_{n}$ 's are approximately the same, indicating both measurements are reliable. The monomer reactivity ratios in the Mayo-Lewis model were determined by the Kelen-Tüdós method. ${ }^{10}$ The $\xi-\eta$ plot by this method was shown in Figure 1, where $r_{1}=0.470$ and $r_{2}=0.381$ were obtained by the least squares method. Although these values are different from the values by Fukuda et al. $\left(r_{1}=0.534, r_{2}=\right.$ $\left.0.393^{4}\right)$, the values obtained in our experiment are used for theoretical calculation. Many pairs of values near our values are reported in literature. ${ }^{11}$ In Figure 2, the relationship between $f_{1}$ and $F_{1}$ together with the composition curve calculated by the MayoLewis equation was shown. The experimental points fitted to the Mayo-Lewis equation.

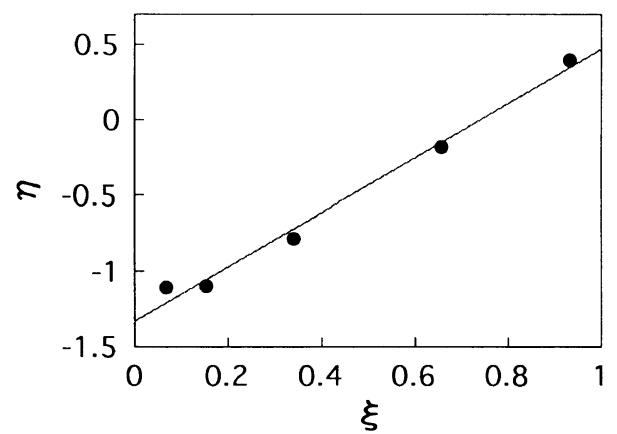

Figure 1. $\xi-\eta$ plot given by the Kelen-Tüdós method.

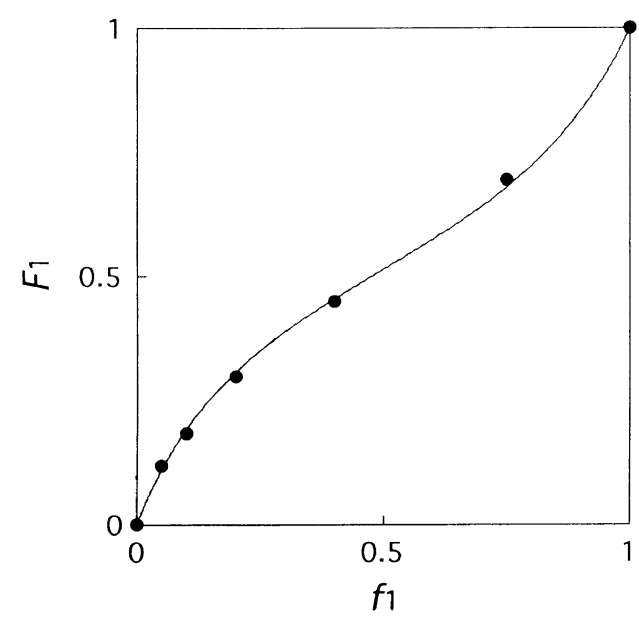

Figure 2. Plot of $F_{1}$ against $f_{1}$; solid curve, calculated from the Mayo-Lewis equation $\left(r_{1}=0.470, r_{2}=0.381\right)$.

The plot of the number-average degree of polymerization $X_{n}$ against $f_{1}$ together with the theoretical curves mentioned later was shown in Figure 3. Here, the $X_{n}$ was calculated from $M_{n}$ by membrane osmometry because membrane osmometry is absolute measurement. In Figure 4, the plot of copolymerization rate $R_{p}$ against $f_{1}$ was shown. The $R_{\mathrm{p}}$ was calculated from

$$
\begin{aligned}
R_{\mathrm{p}} & =\frac{-\mathrm{d}[\mathrm{M}]}{\mathrm{d} t}=\frac{-\Delta[\mathrm{M}]}{\Delta t} \\
& =\frac{C[\mathrm{M}]}{\Delta t} \times \frac{m_{1} f_{1}+m_{2} f_{2}}{m_{1} F_{1}+m_{2} F_{2}}
\end{aligned}
$$

where $\Delta[\mathrm{M}]$ is the variation of the monomer 
Copolymerization Mechanism: Composition and $M_{n}$
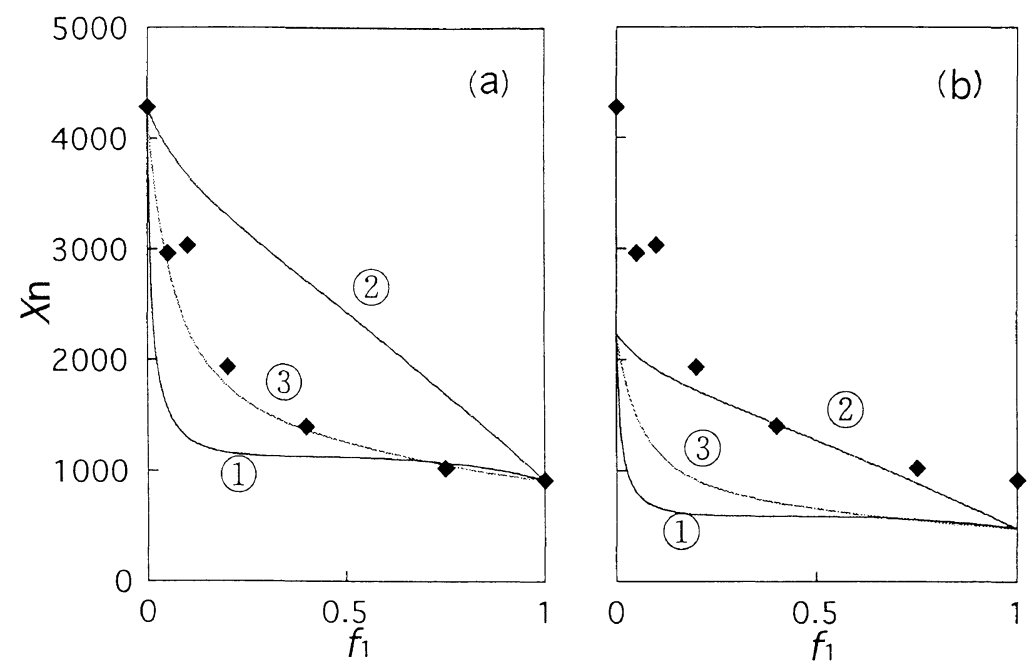

Figure 3. Plot of $X_{n}$ against $f_{1}$ with theoretical curves: (a) calculated by using the experimental value of $2 f^{\prime} k_{\mathrm{d}}$; (b) calculated by using the literature value of $2 f^{\prime} k_{\mathrm{d}}$; (1), (2), (3) see the text.
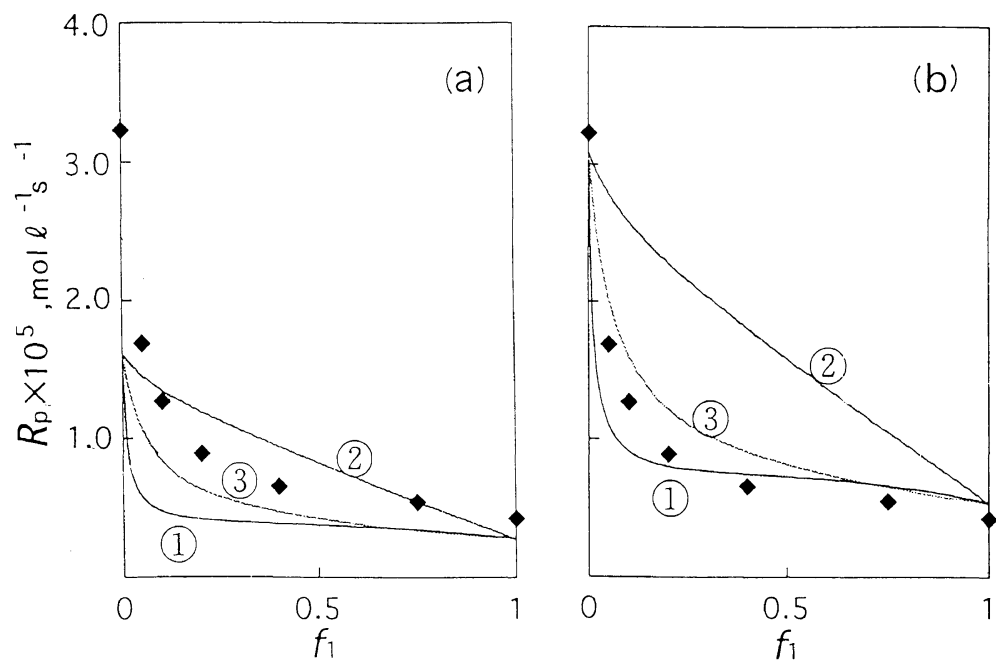

Figure 4. Plot of $R_{n}$ against $f_{1}$ with theoretical curves: (a) calculated by using the experimental value of $2 f^{\prime} k_{\mathrm{d}}$; (b) calculated by using the literature value of $2 f^{\prime} k_{\mathrm{d}}$; (1), (2), (3) see the text.

concentration, $\Delta t$ is the time of copolymerization, $C$ is the conversion (weight fraction), and $m$ is the relative molecular mass of the monomer $\left(m_{1}=100.12\right.$ and $\left.m_{2}=104.15\right)$.

\section{THEORETICAL CALCULATIONS}

According to the polymerization kinetics, $R_{\mathrm{p}}$ and $X_{n}$ are expressed by the following equations as homopolymerization.

$$
\begin{gathered}
R_{\mathrm{p}}=k_{\mathrm{p}}[\mathrm{P} \cdot][\mathrm{M}]=k_{\mathrm{p}}\left\{\left(2 f^{\prime} k_{\mathrm{d}}[\mathrm{I}]\right) / k_{\mathrm{t}}\right\}^{0.5}[\mathrm{M}](7) \\
X_{n}=R_{\mathrm{p}} /\left(\sigma R_{\mathrm{t}}\right)=R_{\mathrm{p}} /\left(\sigma R_{i}\right) \\
\quad=k_{\mathrm{p}}[\mathrm{M}] / \sigma\left(k_{\mathrm{t}} \cdot 2 f^{\prime} k_{\mathrm{d}}[\mathrm{I}]\right)^{0.5}
\end{gathered}
$$

where $k_{\mathrm{p}}$ and $k_{\mathrm{t}}$ are the rate constants of propagation and termination, respectively. $f^{\prime}$ and $k_{\mathrm{d}}$ are the efficiancy and the rate constant 
of decomposition for the initiator. $[\mathrm{P} \cdot]$ and [M] are concentrations of the total radical and the total monomer. $R_{\mathrm{t}}$ and $R_{\mathrm{i}}$ are the rates of temination and initiation. $\sigma$ equals $2-\rho$, where $\rho$ is the fraction of coupling termination.

Generally, $k_{\mathrm{p}}$ and $k_{\mathrm{t}}$ and also $F_{1}$ depend on $f_{1}$. These are functions of $f_{1}$. For the propagation reaction, there are two models, that is, the terminal model and the penultimate model. On the other hand, for the termination reaction, the diffusion-controlled model is accepted recently, though, the reaction was assumed to be chemical-controlled in the classical model.

In this study, $R_{\mathrm{p}}$ and $X_{n}$ were calculated theoretically from eq 7 and 8 by using the following three combinations for the propagation and the termination.
propagation:
termination:
(1) terminal model, chemical controlled (terminal model)
(2) terminal model, diffusion controlled
(3) penultimate model, diffusion controlled

\section{Propagation}

In the binery copolymerization, according to Fukuda et al.,$^{2}$ the equation of $k_{\mathrm{p}}$ given by the Mayo-Lewis model (terminal model) is

$$
k_{\mathrm{p}}=\frac{r_{1} f_{1}^{2}+2 f_{1} f_{2}+r_{2} f_{2}^{2}}{\frac{r_{1} f_{1}}{k_{11}}+\frac{r_{2} f_{2}}{k_{22}}}
$$

where $k_{11}$ and $k_{22}$ are the propagation rate constants of the homopolymerizations, $f_{1}$ and $f_{2}$ are the feed monomer compositions, $r_{1}$ and $r_{2}$ are the monomer reactivity ratios.

In the penultimate model, two propagation rate constants of the homopolymeizations $k_{111}$ and $k_{222}$ are used. The $k_{\mathrm{p}}$ is described by

$$
k_{\mathrm{p}}=\frac{\bar{r}_{1} f_{1}^{2}+2 f_{1} f_{2}+\bar{r}_{2} f_{2}^{2}}{\frac{\bar{r}_{1} f_{1}}{\bar{k}_{11}}+\frac{\bar{r}_{2} f_{2}}{\bar{k}_{22}}}
$$

$$
\begin{gathered}
\bar{r}_{1}=\frac{r_{1}^{\prime}\left(f_{1} r_{1}+f_{2}\right)}{f_{1} r_{1}^{\prime}+f_{2}} \quad \bar{r}_{2}=\frac{r_{2}^{\prime}\left(f_{2} r_{2}+f_{1}\right)}{f_{2} r_{2}^{\prime}+f_{1}} \\
k_{11}=\frac{k_{11}\left(r_{1} f_{1}+f_{2}\right)}{r_{1} f_{1}+f_{2} / s_{1}} \quad \bar{k}_{22}=\frac{k_{222}\left(r_{2} f_{2}+f_{1}\right)}{r_{2} f_{2}+f_{1} / s_{2}}
\end{gathered}
$$

where $r_{i}$ and $s_{i}$ ( $i$ means monomer species) are the monomer reactivity ratios and the radical reactivity ratios, respectively. These reactivity ratios were given by eq 2,3 , and 4 . In the present system, the composition curve fitted well to the Mayo-Lewis equation. The following relation can be assumed.

$$
r_{1}=r_{1}^{\prime}\left(=\bar{r}_{1}\right)
$$

According to Fukuda et al., ${ }^{2,5}$ eq 5 and 14 were assumed.

$$
s_{1}=s_{2}=\left(r_{1} r_{2}\right)^{0.5}
$$

\section{Termination}

For the termination reaction, there are the chemical controlled and the diffusion controlled models. The chemical controlled model is involved in the Walling equation ${ }^{12}$ that describes the copolymerization rate. For the diffusion controlled model, some models are proposed. At present, two models that consider the diffusion motion of the whole molecule are powerful. ${ }^{13,14}$ In this study, the equation proposed by North was used for the theoretical calculations. According to "the North model", ${ }^{13}$

$$
k_{t}=F_{1} k_{\mathrm{t} 1}+F_{2} k_{\mathrm{t} 2}
$$

Actual Calculations The number-average degree of polymerization and the copolymerization rate corresponding to the above combination (1) were calculated by the Walling equation ${ }^{12}$ conventionally. 


$$
\begin{aligned}
R_{\mathrm{p}} & =\frac{R_{i}^{0.5}\left(r_{1}\left[\mathrm{M}_{1}\right]^{2}+2\left[\mathrm{M}_{1}\right]\left[\mathrm{M}_{2}\right]+r_{2}\left[\mathrm{M}_{2}\right]^{2}\right)}{\left(\delta_{1}^{2} r_{1}^{2}\left[\mathrm{M}_{1}\right]^{2}+2 \phi \delta_{1} \delta_{2} r_{1} r_{2}\left[\mathrm{M}_{1}\right]\left[\mathrm{M}_{2}\right]+\delta_{2}^{2} r_{2}^{2}\left[\mathrm{M}_{2}\right]^{2}\right)^{0.5}} \\
& =\frac{R_{i}^{0.5}\left(r_{1} f_{1}^{2}+2 f_{1} f_{2}+r_{2} f_{2}^{2}\right)[\mathrm{M}]}{\left(\delta_{1}^{2} r_{1}^{2} f_{1}^{2}+2 \phi \delta_{1} \delta_{2} r_{1} r_{2} f_{1} f_{2}+\delta_{2}^{2} r_{2}^{2} f_{2}^{2}\right)^{0.5}} \\
X_{n} & =\frac{\left(r_{1} f_{1}^{2}+2 f_{1} f_{2}+r_{2} f_{2}^{2}\right)[\mathrm{M}]}{\sigma R_{i}^{0.5}\left(\delta_{1}^{2} r_{1}^{2} f_{1}^{2}+2 \phi \delta_{1} \delta_{2} r_{1} r_{2} f_{1} f_{2}+\delta_{2}^{2} r_{2}^{2} f_{2}^{2}\right)^{0.5}}
\end{aligned}
$$

where $[\mathrm{M}]=\left[\mathrm{M}_{1}\right]+\left[\mathrm{M}_{2}\right], \delta_{1}=\left(k_{\mathrm{t} 1} / k_{11}^{2}\right)^{0.5}$, $\delta_{1}=\left(k_{\mathrm{t} 2} / k_{22}^{2}\right)^{0.5}, \phi=k_{\mathrm{t} 12} /\left(k_{\mathrm{t} 1} k_{\mathrm{t} 2}\right)^{0.5}, \sigma=2-\rho$. For the monomer reactivity ratios, our experimental values were used (as well as (2) and (3)). In the calculation for the combination (2), equations of 7, 8, 9, and 15 were used. In the calculation for the combination (3), equations of $7,8,10,11,12$, and 15 were used. For the rate constants, the literature values were used, where $k_{11}=k_{111}=116\left(1 \mathrm{~mol}^{-1}\right.$ $\left.\mathrm{s}^{-1}\right),{ }^{4} \quad k_{22}=k_{222}=381 \quad\left(1 \mathrm{~mol}^{-1} \mathrm{~s}^{-1}\right),{ }^{4} k_{\mathrm{t} 1}=$ $108 \times 10^{6}\left(1 \mathrm{~mol}^{-1} \mathrm{~s}^{-1}\right),{ }^{4} k_{t 2}=35 \times 10^{6}\left(1 \mathrm{~mol}^{-1}\right.$ $\left.\mathrm{s}^{-1}\right),{ }^{4} \phi=14 .{ }^{14}$ For $2 f^{\prime} k_{\mathrm{d}}$, the literature ${ }^{4}$ and the experimental values were used. The values of $2 f^{\prime} k_{\mathrm{d}}$ is generally dependent on the feed monomer composition. However, the average value $\left(5.89 \times 10^{-7} \mathrm{~s}^{-1}\right)$ in a literature ${ }^{4}$ was used because the dependency is small. On the other hand, the termination in the homopolymerization of styrene $\left(f_{1}=1\right)$ is known to be the coupling $(\rho=1) .{ }^{15}$ The experimental value of the $2 f^{\prime} k_{\mathrm{d}}\left(1.60 \times 10^{-7} \mathrm{~s}^{-1}\right)$ was calculated from $X_{n}$ of polystyrene $\left(f_{1}=1\right)$ obtained in this study by using eq 8 . The value of $2 f^{\prime} k_{\mathrm{d}}$ thus obtained was used for all over the feed monomer compositions. The $\rho$ was obtained by assumption of the following equation.

$$
\rho=\rho_{1} f_{1}+\rho_{2} f_{2}
$$

where $\rho_{1}$ and $\rho_{2}$ are coupling fractions for termination in the homopolymerization of styrene $\left(f_{1}=1\right)$ and in that of MMA $\left(f_{1}=0\right)$, respectively. The $\rho_{2}$ were calculated by using eq 8 with $X_{n}$ of MMA $\left(f_{1}=0\right)$ and the above value of $2 f^{\prime} k_{\mathrm{d}}$.

\section{DISCUSSION}

In Figure 3 and Figure 4, the relationships between $X_{n}$ and $f_{1}$ and between $R_{\mathrm{p}}$ and $f_{1}$ are illustrated for both of the experimental data and the theoretical curves mentioned above, respectively. In both figures, the theoretical curves in (a) were calcullated by the value of $2 f^{\prime} k_{\mathrm{d}}$ obtained from the experimental $M_{n}$ of polystyrene and the curves in (b) were calculated by the value in literature.

The theoretical curves denoted by (1) are based on the classical copolymerization model. The classical model was used only for the reference because the chemical-controlled termination is low reliabile. ${ }^{14,16}$ Therefore, the model (2) and the new model (3) should be compared with the experimental data.

Qualitatively, in Figure 3 and Figure 4, the curves by the model (2) don't describe the tendencies of $X_{n}$ and $R_{\mathrm{p}}$ varing with $f_{1}$, but the curves by the model (3) describe well tendencies of the experimental data.

Quantitatively, however, the agreements between the experimental data and the theoretical curves are not necessarily good. In order to discuss this point, the experimental data were compared with both theoretical curves calculated by the values of $2 f^{\prime} k_{\mathrm{d}}$ from the present experiment and the literature.

In Figure 3, the experimental relationship between $X_{n}$ and $f_{1}$ is in good agreement with the theoretical curve in (a) but descripant with the theoretical curve in (b). In Figure 4, the calculated curve in (a) is lower, but the curve in (b) is higher than the experimental data. If comparing both cases of (a) and (b) in the 
region of very low $f_{1}$, the curve in (b) is in good agreement with the experimental data, in contradiction to the results for $X_{n}$ (Figure 3).

The reason for the contradiction concerning the value of $2 f^{\prime} k_{\mathrm{d}}$ in Figure 3 and Figure 4 is probably that the molecular weights of samples measured by osmometry are different from those assumed by the kinetics. That is, in the kinetics, the polymer involves even molecules of the oligomer region. Accodingly, the theoretical $M_{n}$, namely $X_{n}$, is the average value of the sample which involves these low molecular weight components, while in the actual copolymer samples the large number of the low molecular weight components are lost by precipitation purification. $M_{n}$ is influenced strongly. by the number of the low molecular weight components, therefore the $M_{n}$ by osmometry is larger than that expected theoretically (kinetically). The experimental value of $2 f^{\prime} k_{\mathrm{d}}$ calculated from $X_{n}$ of polystyrene $\left(f_{1}=1\right)$ becomes smaller than actual value (literature value). However, the theoretical $X_{n}$ calculated by using the experimental value of $2 f^{\prime} k_{\mathrm{d}}$ is in good agreement with the experimental data of $X_{n}$ (Figure 3(a)) because the experimental value of $2 f^{\prime} k_{\mathrm{d}}$ is adjusted by the experimental value of $M_{n}$. On the other hand, the effect of the weight loss on $R_{\mathrm{p}}$ is negligible because the weight loss of the low molecular weight components (oligomer components) by the precipitation purification is small. Therefore, the theoretical curve of $R_{\mathrm{p}}$ calculated by using the lower $2 f^{\prime} k_{\mathrm{d}}$ value obtained experimentally may become lower than the experimental points.
Taking into account that the number loss by the precipitation purification is large whereas the weight loss is small, it may be concluded that the combination of the penultimate model in the propagation and the diffusion-controlled model in the termination (the model (3)) can describe well the relationships for $X_{n}$ and $R_{\mathrm{p}}$.

\section{REFERENCES}

1. F. R. Mayo and F. M. Lewis, J. Am. Chem. Soc., 66, 1954 (1944).

2. T. Fukuda, Y.-D. Ma, and H. Inagaki, Macromolecules, 18, 17 (1985).

3. Y.-D. Ma, T. Fukuda, and H. Inagaki, Macromolecules, 18, 25 (1985).

4. T. Fukuda, K. Kubo, Y.-D. Ma, and H. Inagaki, Polym. J., 19, 523 (1987).

5. Y.-D. Ma, T. Fukuda, and H. Inagaki, Makromol. Chem., Rapid. Commun., 8, 495 (1987).

6. T. Fukuda, Y.-D. Ma, K. Kubo, and A. Takada, Polym. J., 21, 1003 (1989).

7. T. Fukuda, Y.-D. Ma, K. Kubo, and H. Inagaki, Macromolecules, 24, 370 (1991).

8. Y.-D. Ma, Y.-C. Won, K. Kubo, and T. Fukuda, Macromolecules, 26, 6766 (1993).

9. Y.-D. Ma, P.-S. Kim, K. Kubo, and T. Fukuda, Polymer, 35, 1375 (1994).

10. T. Kelen, F. Tüdós, and B. Turcsányi, Polym. Bull., 2, 71 (1980).

11. R.Z. Greenly, "Polymer Handbook," 3rd ed., Wiley, New York, N.Y., 1989.

12. C. Walling, J. Am. Chem. Soc., 71, 1930 (1949).

13. J. N. Atherton and A. M. North, Trans. Faraday Soc., 58, 2049 (1962).

14. T. Fukuda, K. Kubo, and Y.-D. Ma, Prog. Polym. Sci., 17, 875 (1992).

15. J. C. Bevington, H. W. Melville, and R. P. Taylor, J. Polym. Sci., 14, 463 (1954).

16. M. Imoto, "Yukihannou Jyosetu (Introduction of Organic Reaction) Free Radical," Kagaku Dojin, Tokyo, 1976. 